\title{
Editorial \\ Robotic Platforms for Assistance to People with Disabilities
}

\author{
Carlos A. Jara ${ }^{1, *(1)}$ and Juan A. Corrales ${ }^{2}(\mathbb{D}$ \\ 1 Human Robotics Group, University of Alicante, 03690 Alicante, Spain \\ 2 Centro Singular de Investigación en Tecnoloxías Intelixentes (CiTIUS), Universidade de Santiago de Compostela, \\ 15782 Santiago de Compostela, Spain; juanantonio.corrales@usc.es \\ * Correspondence: carlos.jara@ua.es
}

check for

updates

Citation: Jara, C.A.; Corrales, J.A.

Robotic Platforms for Assistance to People with Disabilities. Appl. Sci. 2022, 12, 2235. https://doi.org/ 10.3390/app12042235

Received: 7 February 2022

Accepted: 14 February 2022

Published: 21 February 2022

Publisher's Note: MDPI stays neutral with regard to jurisdictional claims in published maps and institutional affiliations.

Copyright: (c) 2022 by the authors. Licensee MDPI, Basel, Switzerland. This article is an open access article distributed under the terms and conditions of the Creative Commons Attribution (CC BY) license (https:// creativecommons.org/licenses/by/ $4.0 /)$.

\section{Introduction}

People with congenital and/or acquired disabilities constitute a great number of dependents in today's society. These patients lack enough autonomy to live an independent life. Robotic platforms for helping people with disabilities are being developed with the aim of providing both rehabilitation treatment and assistance in improving their quality of life, mainly for those who have mobility problems or some type of functional disability.

A high demand for services of assisted and rehabilitation robotic platforms is expected as a result of the health status of the world due to the COVID-19 pandemic. Currently, according to the WHO (World Health Organization), existing rehabilitation services have been disrupted in $60-70 \%$ of countries due to this pandemic, because of the need to avoid human contact. Therefore, countries must face major challenges to ensure the health and autonomy of their disabled population. Robotic platforms are necessary to ensure assistance and rehabilitation for disabled people in the current global situation.

The capacity of robotic platforms in this area must be continuously improved in order to benefit the healthcare sector in aspects such as chronic disease prevention, assistance, and autonomy. For this reason, research about human-robot interaction in these robotic assistance environments must grow and advance because this topic demands sensitive and intelligent robotic platforms, equipped with complex sensory systems, high handling functionalities, safe control strategies, and intelligent computer vision algorithms. All these technological and scientific developments in different aspects of human-robot interaction can also be extended to other application areas (industry, agriculture, education, etc.) where the assistance of robots is crucial due to physically and/or psychologically demanding tasks.

This Special Issue of Applied Sciences called "Robotic Platforms for Assistance to People with Disabilities" has published seven papers which cover recent advances in the field of robotic platforms to assist disabled people in daily or clinical environments. The papers address innovative solutions in this field, including affordable assistive robotics devices, new techniques in control/computer vision for intelligent and safe human-robot interaction, and advances in mobile manipulators for assistive tasks. These papers represent significant contributions to the research field, which will be summarized in the next section.

\section{Contributions}

Mobile robotic platforms are usually employed to support elderly people in living independently and to assist disabled people in carrying out the activities of daily living independently. In [1], Catalan et al. present a modular mobile robotic platform to assist impaired people based on an upper-limb robotic exoskeleton mounted on a robotized wheelchair. This approach, in comparison with the existing robot assistants, can highlight its modularity and capability to help disabled people with many components: voice-control system, eye-tracking glasses, RGB-D cameras, robotic arm and hand exoskeletons, and a BNCI (brain/neural-computer interaction) system.

In most cases, disabled people must be guided through indoor/outdoor places to their destination, avoiding any obstacles. Specifically, visually impaired people must overcome 
many difficulties to walk safely to their destination. For that reason, robotic platforms for visually impaired people must integrate a suitable communication method to assist them. In these cases, dialogue in natural language is the most important communication method to guide them. In [2], a dialogue system for human-robot communication is proposed based on the knowledge graph in order to provide an accurate destination for the navigation system.

Human pose estimation is a current topic of research, especially for the safety of human-robot collaboration and the evaluation of human biomarkers. In this field, evaluation of low-cost markerless human pose estimators has received much attention for their diversity of applications, especially in rehabilitation robotic environments. In [3], Hernández et al. present an evaluation of the angles in the elbow and shoulder joints, estimated by OpenPose and Detectron 2, during four typical upper-limb rehabilitation exercises: elbow side flexion, elbow flexion, shoulder extension, and shoulder abduction. A low-cost setup of two Kinect 2 RGB-D cameras was used to obtain the ground truth of the joint and skeleton estimations during the different exercises.

Direct physical assistance from robotic systems can be useful for the rehabilitation of damaged limbs in accidents, or for the prevention of musculoskeletal problems and fatigue in repetitive tasks. Two main approaches have been developed in the literature: exoskeletons that are tightened to the user's body in order to move their limbs directly, and collaborative robots that handle the tools that have to be used by the user in physically demanding tasks. Two main techniques are used for controlling these systems: by interpreting brain signals so that the user's intentions are estimated, or by including force/torque feedback so that the force applied by the user in a tool is processed and amplified. In [4], Ferrero et al. use an EEG (electroencephalogram) cap for controlling a lower-limb exoskeleton based on two phases (training and tests) applied in walking and standing conditions. In [5], Maithani et al. use an impedance control strategy that calculates the force applied by a user in a tool (e.g., a knife), attached to a collaborative robotic arm for meat-cutting applications. They include an intent prediction module in order to reduce the forces applied by the user by $20 \%$, regarding classical force amplification strategies.

In addition to the previous direct physical assistance solutions, rehabilitation applications can also benefit from sensor-based monitoring systems that have lower costs and thus can be used widely, not only in hospitals but also at home. Gomez-Donoso et al. in [6] propose a new software platform that is able to analyze data coming from a low-cost hand-tracking device and a low-cost surface electromyography (sEMG) sensor, in order to verify that a set of rehabilitation exercises is carried out competently. This software is implemented in a social robot in order to improve the engagement of the patients and to improve feedback about the therapy.

The use of monitoring devices can also be applied to neurological disorders in order to improve the living conditions of people suffering from them. In [7], Vicente-Samper et al. propose mixing data from a personal device (that measures motor activity with an inertial sensor, heart rate, and body temperature) with that from an environmental device (based on a camera for tracking people around the user in order to detect social interactions) into a standard database where machine learning algorithms can extract user models (i.e., concentration level—distracted vs. focused — of the user while performing a task, such as reading a book).

Conflicts of Interest: The authors declare no conflict of interest.

\section{References}

1. Catalan, J.M.; Blanco, A.; Bertomeu-Motos, A.; Garcia-Perez, J.V.; Almonacid, M.; Puerto, R.; Garcia-Aracil, N. A Modular Mobile Robotic Platform to Assist People with Different Degrees of Disability. Appl. Sci. 2021, 11, 7130. [CrossRef]

2. Chen, C.H.; Shiu, M.F.; Chen, S.H. Use Learnable Knowledge Graph in Dialogue System for Visually Impaired Macro Navigation. Appl. Sci. 2021, 11, 6057. [CrossRef]

3. Hernández, Ó.G.; Morell, V.; Ramon, J.L.; Jara, C.A. Human Pose Detection for Robotic-Assisted and Rehabilitation Environments. Appl. Sci. 2021, 11, 4183. [CrossRef] 
4. Ferrero, L.; Quiles, V.; Ortiz, M.; Iáñez, E.; Azorín, J.M. A BMI Based on Motor Imagery and Attention for Commanding a Lower-Limb Robotic Exoskeleton: A Case Study. Appl. Sci. 2021, 11, 4106. [CrossRef]

5. Maithani, H.; Corrales Ramon, J.A.; Lequievre, L.; Mezouar, Y.; Alric, M. Exoscarne: Assistive Strategies for an Industrial Meat Cutting System Based on Physical Human-Robot Interaction. Appl. Sci. 2021, 11, 3907. [CrossRef]

6. Gomez-Donoso, F.; Escalona, F.; Nasri, N.; Cazorla, M. A Hand Motor Skills Rehabilitation for the Injured Implemented on a Social Robot. Appl. Sci. 2021, 11, 2943. [CrossRef]

7. Vicente-Samper, J.M.; Avila-Navarro, E.; Esteve, V.; Sabater-Navarro, J.M. Intelligent Monitoring Platform to Evaluate the Overall State of People with Neurological Disorders. Appl. Sci. 2021, 11, 2789. [CrossRef] 